Research Article

\title{
Output Voltage Control of MMC-Based Microgrid Based on Voltage Fluctuation Compensation Sliding Mode Control
}

\author{
Sheng Xue $\mathbb{D}^{D}$, Xinggui Wang $(\mathbb{D}$, and Xiaoying Li \\ School of Electrical and Information Engineering, Lanzhou University of Technology, Lanzhou, China \\ Correspondence should be addressed to Sheng Xue; xueshenglut@126.com and Xinggui Wang; wangxg689@126.com
}

Received 17 July 2020; Revised 31 July 2020; Accepted 1 August 2020; Published 20 August 2020

Academic Editor: Yang Li

Copyright (c) 2020 Sheng Xue et al. This is an open access article distributed under the Creative Commons Attribution License, which permits unrestricted use, distribution, and reproduction in any medium, provided the original work is properly cited.

\begin{abstract}
As a novel topology of microgrid, the output voltage control of MMC half bridge series microgrid (MMC-MG) is rarely studied. In this paper, on the basis of fully analyzing the mechanism of output voltage fluctuation of MMC-MG under the condition of islanded mode, a control strategy of a hybrid energy storage system is proposed to reduce the generating module (GM) DC-link voltage fluctuation caused by the randomness of renewable energy microsource output power. Moreover, in order to further improve the stabilization of the MMC-MG output voltage and meet the requirements of fast voltage recovery and antijamming, a sliding mode controller is designed. Then, a voltage fluctuation compensation controller is designed to suppress the DC component and fundamental frequency deviation of system output voltage caused by GM DC-link voltage fluctuation. The proposed control approach is validated against simulations using MMC-MG models with 4-GM per arm. The results show that the proposed hybrid energy storage control strategy can suppress the GM DC-link voltage fluctuation, the sliding mode controller can stabilize the system output voltage when the load drastic changes, and the fluctuation compensation strategy can suppress the DC component and the fundamental frequency deviation of system output voltage.
\end{abstract}

\section{Introduction}

Microgrid (MG) is an autonomous system that can achieve self-control, protection, and management, which includes distributed generation (DG), load, energy storage devices, and monitoring and protection devices [1-3]. For the isolated ac MG, the system output voltage loses the power grid support, and effective control methods are needed to maintain the frequency and amplitude of the system output voltage within the standard range [4-6]. The P-Q control algorithm is adopted for renewable energy source (RES) inverters to improve the output power utilization, and the $\mathrm{V}$ - $\mathrm{f}$ control algorithm is adopted for nonrenewable energy source inverters to ensure the stabilization of system voltage and frequency $[7,8]$.

A novel MG topology based on modular multilevel converter half-bridge series structure (MMC-MG) is proposed in [9], and the islanded system output characteristics is analyzed. Different from the multi-inverter parallel ac MG, the output voltage of MMC-MG is superposed by the output voltage of each generating module (GM). Affected by the randomness of the microsource (MS) output power, the DClink voltage of GM fluctuates, which leads to the amplitude fluctuation of MMC-MG output voltage and the increase of harmonic. Therefore, in order to realize the output voltage stable control of MMC-MG, it is necessary to realize the GM DC-link voltage stable control and then realize the output voltage closed-loop control of the system inverter.

The hybrid energy storage system (HESS) composed of the supercapacitor (SC) and battery can be used in MG. It plays an important role in stability control, power quality improvement, and uninterrupted power supply of MG [10]. A new chance constrained programming-based scheduling model is proposed in [11], which makes full use of ESS to provide spinning reserve services for isolated MG. A multiagent sliding mode control strategy for state of charge balancing between distributed DC-microgrid battery energy storage systems is proposed in [12]. A supervisory power management system for a hybrid MG is proposed in [13]; when MG in the islanded mode, the HESS can effectively 
suppress the frequency and voltage fluctuation of the system. For the photovoltaic (PV) MG, a control strategy of virtual synchronous generator (VSG) which combines energy storage and photovoltaic technology is proposed in [14] to stabilize the fluctuation of PV power and improve the frequency stability. Fang et al. [15] proposed a battery/SC HESS to implement VSGs, which is used to achieve frequency regulation for RES in islanded MG. A converter topology to interface a RES with a HESS in a MG is investigated in [16], and the proposed control strategy can improve the power quality and stability. However, the research works mentioned above are mainly concentrated on HESS control for the AC MG with multiple MS inverters connected in parallel, while the HESS for MMC-MG is seldom studied because of its series structure.

For the problem of output voltage closed-loop control of the MS inverter, a robust virtual inertia control for islanded MG is proposed in [17], which enhancing the robust performance and stability of the MG during contingencies. A novel framework of coordinated voltage and frequency control strategy for islanded $\mathrm{MG}$ is studied in [18], the proposed control strategy improves the voltage and frequency regulation, transient response, and MG stability. A control scheme for distributed energy resource based on sliding mode control (SMC) is proposed in [19], which provides fast and stable control on the voltage and frequency of islanded MG. A higher order SMC strategy is studied for islanded $\mathrm{MG}$ to chattering alleviation and improve the system stability in [20]. However, the method studied in the above literature is only applicable to ordinary MG with parallel inverters, not to MMC-MG with microsource inverters in series.

A control scheme of output and circulating current of MMC using sliding mode control (SMC) is proposed in [21], while suppressing second harmonics contents in circulating current. For the MMC system, the voltage of each submodule is supported by the system common DC bus. However, for MMC-MG, there is no common DC-bus voltage supported, and the voltage of each GM is generated by MS. Due to its series structure, the voltage deviation of each GM will cause the system output voltage contain DC component and fundamental deviation, which need to be suppressed.

However, the previous research on SMC for MMC was aimed at the control of output current and circulating current, rather than the output voltage control of the island system. And its control algorithm cannot eliminate the influence of GM voltage deviation on the output voltage of MMC-MG.

In this paper, in order to solve the problem of output voltage control of MMC-MG in the islanded mode, a HESS control strategy suitable for GM DC-link voltage control is proposed. To further stabilize the system output voltage, the feedback linearization is used to decouple the system precisely, and the SMC strategy based on voltage fluctuation compensation(VFC) is designed for the decoupled system. Simulation results of an MMC-MG system with 4-GM per arm is obtained to verify the effectiveness of the proposed strategy. The main contributions of this paper are listed as follows:

(1) The MMC-MG studied in this paper is a new type of MG, which has the advantages of higher output voltage levels and less harmonics than the ordinary MG with multiple inverters in parallel

(2) A SMC strategy for MMC-MG output voltage control is proposed, which meets the requirements of islanding system for fast voltage recovery and antiinterference antijamming

(3) The controller of GM voltage fluctuation compensation is designed to reduce the influence of GM voltage fluctuation on MMC-MG output voltage

The rest of this paper is organized as follows. Section 2 analyses the voltage fluctuation factors of MMC-MG output ac side and GM DC link. Section 3 focuses on the control strategy of HESS. Section 4 presents the modeling and decoupling of the system inverter, including topology models and mathematic models, and state feedback linearization decoupled. Section 5 presents design of the system output controller based on SMC. Simulation of the proposed architecture and control strategy is shown in Section 6 . Conclusions are given in Section 7.

\section{MMC-MG Output Voltage Analysis}

The MMC-MG topology is shown in Figure 1, where the GM is a power generation unit composed of MS, AC/DC or DC/ DC converter, HESS, and half-bridge converter (HC).

The MS in GM can be categorized into renewable energy sources such as wind turbine and PV and nonrenewable energy sources such as diesel generator and microgas turbine generator. And the HESS is only equipped in GM with RES. With GM as the basic module, the three-phase inverter is constructed according to MMC structure, connected to the ac bus through the filter, then connected to the public power grid with a static transfer switch (STS), and the local load is connected to the ac bus. The system is composed of two arms $z(z \in\{\mathrm{p}, n\})$ in each phase $X(X \in\{\mathrm{A}, \mathrm{B}, \mathrm{C}\})$. Each arm is constituted by $N$ series-connected GMs with a series inductor $L$.

Compared with the parallel inverters ac MG, the MMCMG series structure inverter makes the stable of frequency higher. The multilevel output voltage of the system is formed by the superposition of several GM output voltages, which reduces the pollution of harmonics to the power grid. At the same output voltage level, the operating voltage of the MS inverter is reduced, then save the investments of power electronic equipment. Increasing GM can improve the system output voltage level and provide a new grid structure for low and medium voltage MG.

2.1. Inverter Output Voltage Analysis. Ignoring the high harmonics, the output voltage $\mathrm{u}_{\mathrm{AB}}$ of the inverters in the islanded mode under the phase-shifted PWM (PSC-PWM) can be given by 


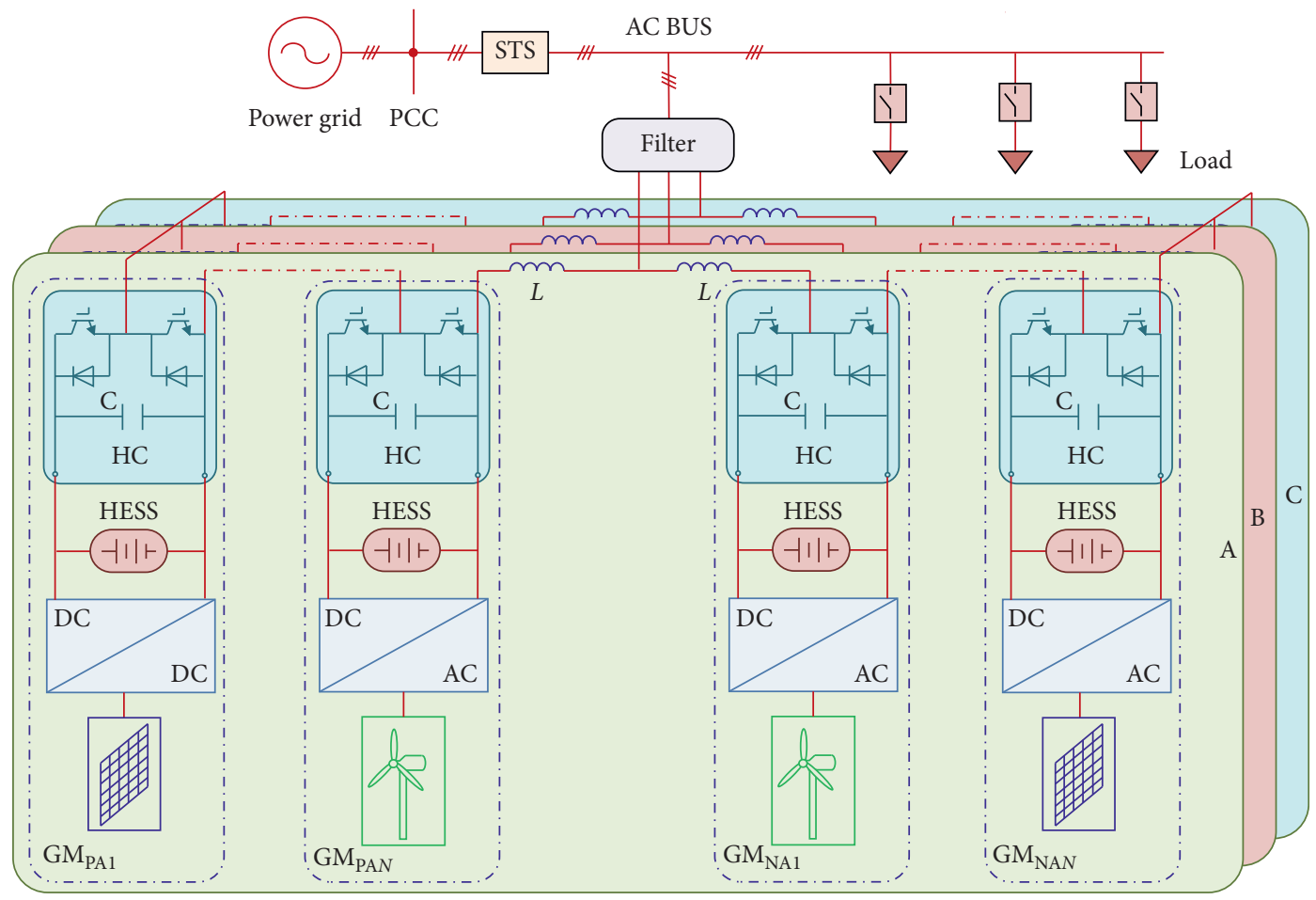

Figure 1: MMC-MG topology structure.

$$
u_{\mathrm{AB}}=u_{\mathrm{ZAB}}+u_{\mathrm{SAB}}-\frac{\sqrt{3} N u_{\mathrm{dc}}^{*}}{2} M \sin \left(\omega_{0} t+\frac{\pi}{6}\right),
$$

where $u_{Z A B}$ is the output DC component, which satisfies

$$
u_{Z \mathrm{AB}}=\frac{1}{4}\left[\Delta u_{p \mathrm{~A}}-\Delta u_{n \mathrm{~A}}+\Delta u_{n \mathrm{~B}}-\Delta u_{p \mathrm{~B}}\right]
$$

where $u_{S A B}$ is the fundamental frequency deviation, which is formed by superimposing two sine waves with the same frequency and different amplitude of the line voltage fundamental component, which satisfies

$$
\begin{aligned}
u_{S \mathrm{AB}}= & \frac{M}{4}\left[\left(\Delta u_{n \mathrm{~B}}+\Delta u_{p \mathrm{~B}}\right) \sin \left(\omega_{0} t-\frac{2 \pi}{3}\right)\right. \\
& \left.-\left(\Delta u_{n \mathrm{~A}}+\Delta u_{p \mathrm{~A}}\right) \sin \omega_{0} t\right],
\end{aligned}
$$

where $M(0 \leq M \leq 1)$ denotes the modulation depth, $\omega_{0}$ is the angular frequency of the output voltage, $u_{\mathrm{dc}}^{*}$ is the voltage reference value of GM DC-link, $\Delta u_{p X_{i}}, \Delta u_{n X_{i}}(i=1,2, \ldots, N)$ are the deviation of GM DC-link voltage and $u_{\mathrm{dc}}^{*}$ in each arm when MMC-MG operation, and $\Delta u_{p X}=\sum_{i=1}^{N} \Delta u_{p X_{i}}$, $\Delta u_{n X}=\sum_{i=1}^{N} \Delta u_{n X_{i}}$.

According to (1)-(3), the voltage deviation of each GM DC link is superimposed on each other, resulting in the output voltage of the MMC-MG including DC component and fundamental frequency deviation.

2.2. GM DC-Link Voltage Fluctuation Analysis. As shown in Figure 2, the ith GM internal currents of phase A positive arm are used for analysis. $i_{\mathrm{g}}$ is the output current of MS, $i_{\mathrm{c}}$ is the charge current of capacitor $C, i_{\mathrm{m}}$ is the input current of $\mathrm{HC}, i_{p \mathrm{~A}}$ is the arm current, and $u_{p A_{i}}$ is the output voltage of

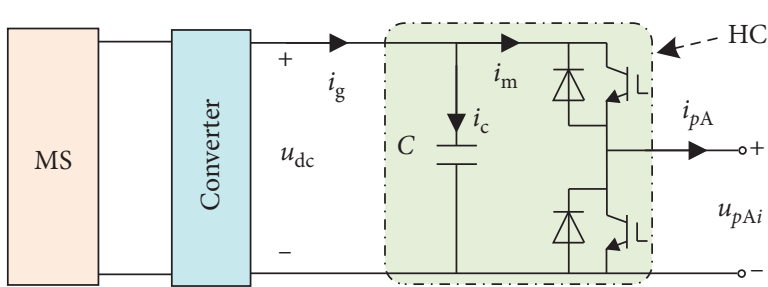

FIGURE 2: Schematic diagram of GM.

HC. When GM is switched on, $i_{\mathrm{m}}=i_{p \mathrm{~A}}$ and $u_{p A_{i}}=u_{\mathrm{dc}}$. When GM is switched off, $i_{\mathrm{m}}=0$ and $u_{p A_{i}}=0$.

MS output power $P_{\mathrm{M}}$ and GM output power $P_{\mathrm{G}}$ can be modeled as follows:

$$
\begin{aligned}
& P_{\mathrm{M}}=u_{\mathrm{dc}} i_{\mathrm{g}}, \\
& P_{\mathrm{G}}=u_{p \mathrm{~A}_{i}} i_{p \mathrm{~A}}=u_{\mathrm{dc}} i_{\mathrm{m}} .
\end{aligned}
$$

The system is cascaded structure, GM is not directly connected to the load, so $P_{\mathrm{G}}$ is called equivalent load power. Then, the GM internal power satisfies

$$
P_{\mathrm{M}}-P_{\mathrm{G}}=u_{\mathrm{dc}} C \frac{d u_{\mathrm{dc}}}{d t}=P_{\mathrm{C}}
$$

where $P_{\mathrm{C}}$ is capacitive $C$ absorption power. Within $t$ to $t+\Delta t$, the variation of GM DC-link voltage satisfies

$$
\Delta u_{\mathrm{dc}}=\sqrt{\frac{2}{C} \int_{t}^{t+\Delta t}\left(P_{\mathrm{M}}-P_{\mathrm{G}}\right) d t}
$$

To keep the GM DC-link voltage stable, $\Delta u_{\mathrm{dc}}$ must to be 0 , that means MS output power should be follow the 
equivalent load power variation. While the output power of MS such as wind turbine and PV are random, it is difficult to maintain the stable of the GM DC-link voltage by means of control. So, it is necessary to add HESS in GM to dynamically adjust the output power and keep voltage stable.

\section{GM DC-Link Voltage Control}

Different from the full-bridge MS inverter used in ordinary AC MG, the GM uses a half-bridge converter. During the switching process, $i_{\mathrm{m}}$ is affected by the arm current, which contains high harmonic content and AC component.

3.1. GM Internal Power Analysis. The HESS is composed of batteries and SC and connected to GM DC link in parallel by bidirectional DC/DC converters. This topology can give full play to their advantages in energy storage. Battery has the advantages of high energy density, which is suitable for regulating low-frequency power fluctuation. SC has the advantages of high power density and fast charge-discharge response, which is suitable for regulating high-frequency power fluctuation [22]. The structure of HESS is shown in Figure 3 , where $u_{\mathrm{sc}}$ and $i_{\mathrm{sc}}$ are the SC voltage and output current, $i_{\mathrm{b}}$ is the battery output current, $u_{\mathrm{dc}}$ is GM DC-link voltage, and $\mathrm{BC}_{1}$ and $\mathrm{BC}_{2}$ are the bidirectional $\mathrm{DC} / \mathrm{DC}$ converters.

When GM is switched on, the internal power is satisfied:

$$
P_{\mathrm{M}}-P_{\mathrm{G}}-P_{\mathrm{F}}=P_{\mathrm{C}} \text {, }
$$

where $P_{\mathrm{F}}$ is the absorption energy of HESS, which is used to suppress the GM DC-link voltage fluctuation caused by fluctuation of MS output power and arm current. When the DC-link voltage is stable, $P_{\mathrm{C}}=0$, and (7) should be rewritten as follows:

$$
P_{\mathrm{M}}-P_{\mathrm{G}}=P_{\mathrm{F}}
$$

$P_{\mathrm{F}}$ is caused by the MS output power fluctuation and the equivalent load change. When the HESS is used to smooth power fluctuation, $P_{\mathrm{F}}$ is divided into a low-frequency fluctuation $P_{\mathrm{L}}$ and a high-frequency fluctuation $P_{\mathrm{H}}$. The SC mainly absorb or release the $P_{\mathrm{H}}$, that can reducing the response frequency of the battery to the $P_{\mathrm{H}}$, then reducing the number of charges and discharges of battery, and improving HESS service life. $P_{\mathrm{L}}$ and $P_{\mathrm{H}}$ can be expressed as follows:

$$
\left\{\begin{array}{l}
P_{\mathrm{L}}=P_{\mathrm{F}} \frac{1}{\tau_{1} s+1}, \\
P_{\mathrm{H}}=P_{\mathrm{F}}-P_{\mathrm{L}},
\end{array}\right.
$$

where $s$ is the differential operator, $1 /\left(\tau_{1} s+1\right)$ is a low-pass filter (LPF), and $\tau_{1}$ is a time constant.

3.2. Hybrid Energy Storage System Control. The SC operating voltage is divided into the following five intervals [23]:

(1) High voltage over-limit zone (HOZ): $u_{\mathrm{sc}}>u_{\mathrm{sc}-\max }$

(2) High voltage limit zone (HLZ): $u_{\mathrm{sc}-\mathrm{h}}<u_{\mathrm{sc}}<u_{\mathrm{sc}-\max }$

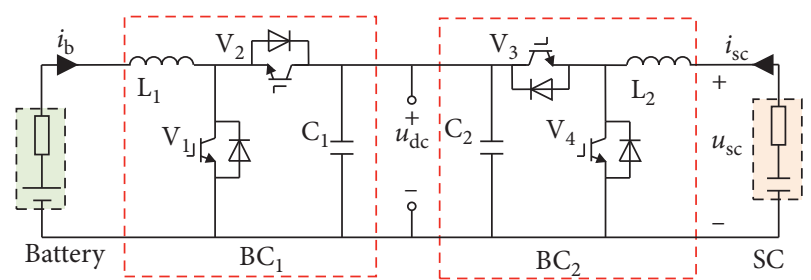

FIgURE 3: Structure diagram of HESS.

(3) Normal working zone (NZ): $u_{\mathrm{sc}-1}<u_{\mathrm{sc}}<u_{\mathrm{sc}-\mathrm{h}}$

(4) Low voltage limit zone (LLZ): $u_{\mathrm{sc}-\min }<u_{\mathrm{sc}}<u_{\mathrm{sc}-1}$

(5) Low voltage over-limit zone (LOZ): $u_{\mathrm{sc}}<u_{\mathrm{sc}-\min }$

$u_{\mathrm{sc}-\max }$ and $u_{\mathrm{sc}-\min }$ are the maximum voltage and minimum voltage of SC and $u_{\mathrm{sc}-\mathrm{h}}$ and $u_{\mathrm{sc}-1}$ are the upperlimit value and lower-limit value of the SC voltage controller. In order to prevent overcharge and discharge of the SC, it is necessary to correct the charge and discharge power according to the voltage. The power correction value $\Delta P_{\mathrm{sc}}$ in the five working intervals is shown in Table 1.

The battery bidirectional DC/DC converter operates in a single-ended regulated mode to ensure that the GM DC-link voltage is stable, and the power correction value of battery is $-\Delta P_{\mathrm{sc}}$. The control diagram of the HESS is shown in Figure 4 , and PC is the power correction controller based on Table 1.

\section{Modeling and Decoupling of Inverter}

4.1. Mathematical Model of System Inverter. As showN in Figure 5, the equivalent circuit diagram of the MMC-MG inverter in the islanded mode is used for analysis.

In Figure $5, L_{f}$ is the filter inductor, $C_{f}$ is the filter capacitor, $L_{\mathrm{am}}$ is the arm equivalent inductor and $L_{\mathrm{am}}=L / 2, i_{\mathrm{X}}$ is the phase current, $u_{\mathrm{X}}$ is the output voltage of phase $X, u_{\mathrm{oX}}$ is the filter capacitor voltage, $R_{\text {load }}$ is the load, and $R$ is the arm equivalent resistance.

According to KCL (Kirchhoff's current law) and KVL (Kirchhoff's voltage law), the voltage-to-current equation of the inverter in dq0 coordinate can be expressed as follows:

$$
\begin{aligned}
& u_{\mathrm{d}}=\frac{R}{2} i_{\mathrm{d}}+L_{m} \frac{d i_{\mathrm{d}}}{d t}-\omega_{0} L_{m} i_{\mathrm{q}}+u_{\mathrm{od}}, \\
& i_{\mathrm{d}}=C_{f} \frac{d u_{\mathrm{od}}}{d t}-\omega_{0} C_{f} u_{\mathrm{q}}+i_{\mathrm{od}}, \\
& u_{\mathrm{q}}=\frac{R}{2} i_{\mathrm{q}}+L_{m} \frac{d i_{\mathrm{q}}}{d t}+\omega_{0} L_{m} i_{\mathrm{d}}+u_{\mathrm{oq}},
\end{aligned}
$$

$$
i_{\mathrm{q}}=C_{f} \frac{d u_{\mathrm{oq}}}{d t}+\omega_{0} C_{f} u_{\mathrm{d}}+i_{\mathrm{oq}}
$$

where $u_{\mathrm{d}}$ and $i_{\mathrm{d}}$ are the active components of the phase voltage and current and $u_{\mathrm{q}}$ and $i_{\mathrm{q}}$ are the reactive components of the phase voltage and current. Similarly, $u_{\text {od }}, i_{\text {od }}$ and $u_{\mathrm{oq}}, i_{\mathrm{oq}}$ are the active and reactive components of the 
TABLe 1: Power correction value $\Delta P_{\mathrm{sc}}$.

\begin{tabular}{lcc}
\hline Intervals & $\Delta P_{\mathrm{sc}}\left(P_{\mathrm{H}}>0\right)$ & $\Delta P_{\mathrm{sc}}\left(P_{\mathrm{H}}<0\right)$ \\
\hline HOZ & 0 & $-P_{\mathrm{H}}$ \\
HLZ & $u_{\mathrm{sc}}-u_{\mathrm{sc}-\mathrm{h}} / u_{\mathrm{sc}-\max }-u_{\mathrm{sc}-\mathrm{h}} P_{\mathrm{H}}$ & $u_{\mathrm{sc}-\mathrm{h}}-u_{\mathrm{sc}} / u_{\mathrm{sc}-\mathrm{max}}-u_{\mathrm{sc}-\mathrm{h}} P_{\mathrm{H}}$ \\
NZ & 0 & 0 \\
LLZ & $u_{\mathrm{sc}}-u_{\mathrm{sc}-1} / u_{\mathrm{sc}-1}-u_{\mathrm{sc}-\min } P_{\mathrm{H}}$ & $u_{\mathrm{sc}-1}-u_{\mathrm{sc}} / u_{\mathrm{sc}-1}-u_{\mathrm{sc}-\mathrm{min}} P_{\mathrm{H}}$ \\
LOZ & $-P_{\mathrm{H}}$ & 0 \\
\hline
\end{tabular}

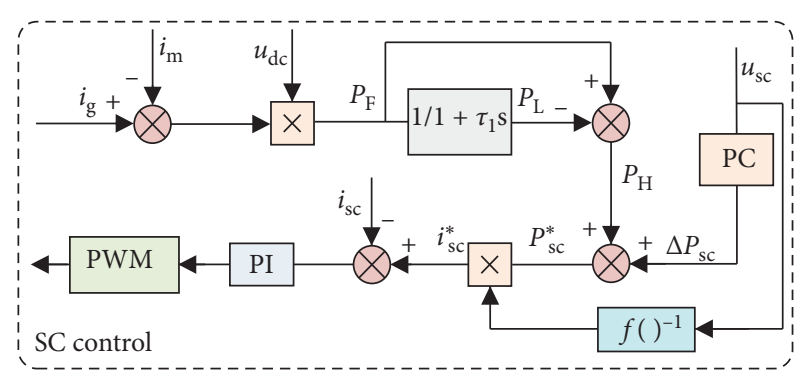

(a)

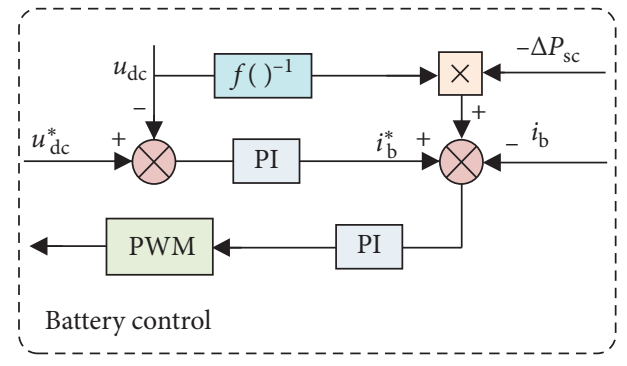

(b)

FIGURE 4: Block diagram of energy storage converter control.

capacitor voltage and load current. $L_{\mathrm{m}}$ is the sum of the $L_{f}$ and $L_{\mathrm{am}}$, and $\omega_{0}$ is the voltage angular frequency.

4.2. State Feedback Linearization Decoupled. The voltage-tocurrent equations under dq0 coordinate of the inverter can rewritten as follows:

$$
\frac{d}{d t}\left[\begin{array}{l}
i_{\mathrm{d}} \\
i_{\mathrm{q}}
\end{array}\right]=\left[\begin{array}{cc}
\frac{-R}{2 L_{m}} & \omega_{0} \\
-\omega_{0} & \frac{-R}{2 L_{m}}
\end{array}\right]\left[\begin{array}{l}
i_{\mathrm{d}} \\
i_{\mathrm{q}}
\end{array}\right]+\left[\begin{array}{c}
\frac{u_{\mathrm{d}}-u_{\mathrm{od}}}{L_{m}} \\
\frac{u_{\mathrm{q}}-u_{\mathrm{oq}}}{L_{m}}
\end{array}\right] .
$$

In order to improve the decoupling accuracy, the state feedback linearization (SFL) method is used for system decoupling. Take state variables as $x=\left[i_{\mathrm{d}} i_{\mathrm{q}}\right]^{\mathrm{T}}$, input variables as $u=\left[\begin{array}{ll}u_{\mathrm{d}} & u_{\mathrm{q}}\end{array}\right]^{\mathrm{T}}$, and output variables as $y=\left[\begin{array}{ll}h_{1}(x) & h_{2}(x)\end{array}\right]^{\mathrm{T}}$, where $h_{1}(x)=i_{\mathrm{d}}, h_{2}(x)=i_{\mathrm{q}}$. The state equation of affine 2input 2-output nonlinear system is obtained as follows:

$$
\left\{\begin{array}{l}
\dot{x}=f(x)+g_{1}(x) u_{1}+g_{2}(x) u_{2} \\
y_{1}=h_{1}(x) \\
y_{2}=h_{2}(x)
\end{array}\right.
$$

where $\quad g_{1}(x)=\left[\begin{array}{c}1 / L_{m} \\ 0\end{array}\right], \quad g_{2}(x)=\left[\begin{array}{c}0 \\ 1 / L_{m}\end{array}\right]$, and $f(x)=\left[\begin{array}{l}-R / 2 L_{m} x_{1}+\omega_{0} x_{2}-u_{\mathrm{od}} / L_{m} \\ -\omega_{0} x_{1}-R / 2 L_{m} x_{2}-u_{\mathrm{oq}} / L_{m}\end{array}\right]$.

The solvable condition of the system full-state feedback linearization problem is that the system has relative degree $\rho 1, \rho 2$ and satisfies $\rho 1+\rho 2=n$, where $n$ is the dimension of the system variables. According to the relative degree definition, there is any point $x^{0}$ of the state variable $x$ satisfied:

$$
A\left(x^{0}\right)=\left[\begin{array}{cc}
L_{g_{1}} L_{f}^{0} h_{1}\left(x^{0}\right) & L_{g_{2}} L_{f}^{0} h_{1}\left(x^{0}\right) \\
L_{g_{1}} L_{f}^{0} h_{2}\left(x^{0}\right) & L_{g_{2}} L_{f}^{0} h_{2}\left(x^{0}\right)
\end{array}\right],
$$

where $L_{f}^{0} h(x)=h(x), L_{g_{j}} h_{i}(x)$ is the Lie derivative of $h(x)$ for $g(x)$ and $L_{g_{j}} h_{i}(x)=\partial h_{i}(x) / \partial x g_{j}(x), \quad(i, j=1,2)$.

Then, matrix $A$ is

$$
A\left(x^{0}\right)=\left[\begin{array}{cc}
1 / L_{m} & 0 \\
0 & 1 / L_{m}
\end{array}\right] .
$$

Based on (14), the relative degree of system $\rho_{1}=\rho_{2}=1$ and $\rho_{1}+\rho_{2}=n=2$. The system satisfies the conditions of exact linearization. Exact linearization decoupling of the system, constructing a new system input variable $v$, there are matrix $A(x), b(x)$, and the input variable $v$ satisfies

$$
v=b(x)+A(x) u
$$

where $b(x)=\left[\begin{array}{l}L_{f} h_{1}(x) \\ L_{f} h_{2}(x)\end{array}\right]=\left[\begin{array}{l}-R / 2 L_{m} x_{1}+\omega_{0} x_{2}-u_{\mathrm{oq}} / L_{m} \\ -\omega_{0} x_{1}-R / 2 L_{m} x_{2}-u_{\mathrm{oq}} / L_{m}\end{array}\right]$ and $A(x)=\left[\begin{array}{ll}L_{g_{1}} h_{1}(x) & L_{g_{2}} h_{1}(x) \\ L_{g_{1}} h_{2}(x) & L_{g_{2}} h_{2}(x)\end{array}\right]=\left[\begin{array}{cc}1 / L_{m} & 0 \\ 0 & 1 / L_{m}\end{array}\right]$.

Then, the relationship between new input variables and output variables is satisfied:

$$
\left[\begin{array}{l}
v_{1} \\
v_{2}
\end{array}\right]=b(x)+A(x) u=\left[\begin{array}{l}
\dot{y}_{1} \\
\dot{y}_{2}
\end{array}\right]
$$

It can be seen from (16) that the system is decoupled into a first-order linear system. Then, the system becomes a typical tracking system, and the control target is to track the given values $i_{d}^{*}$ and $i_{q}^{*}$ of the system output variables $i_{\mathrm{d}}$ and $i_{\mathrm{q}}$. 


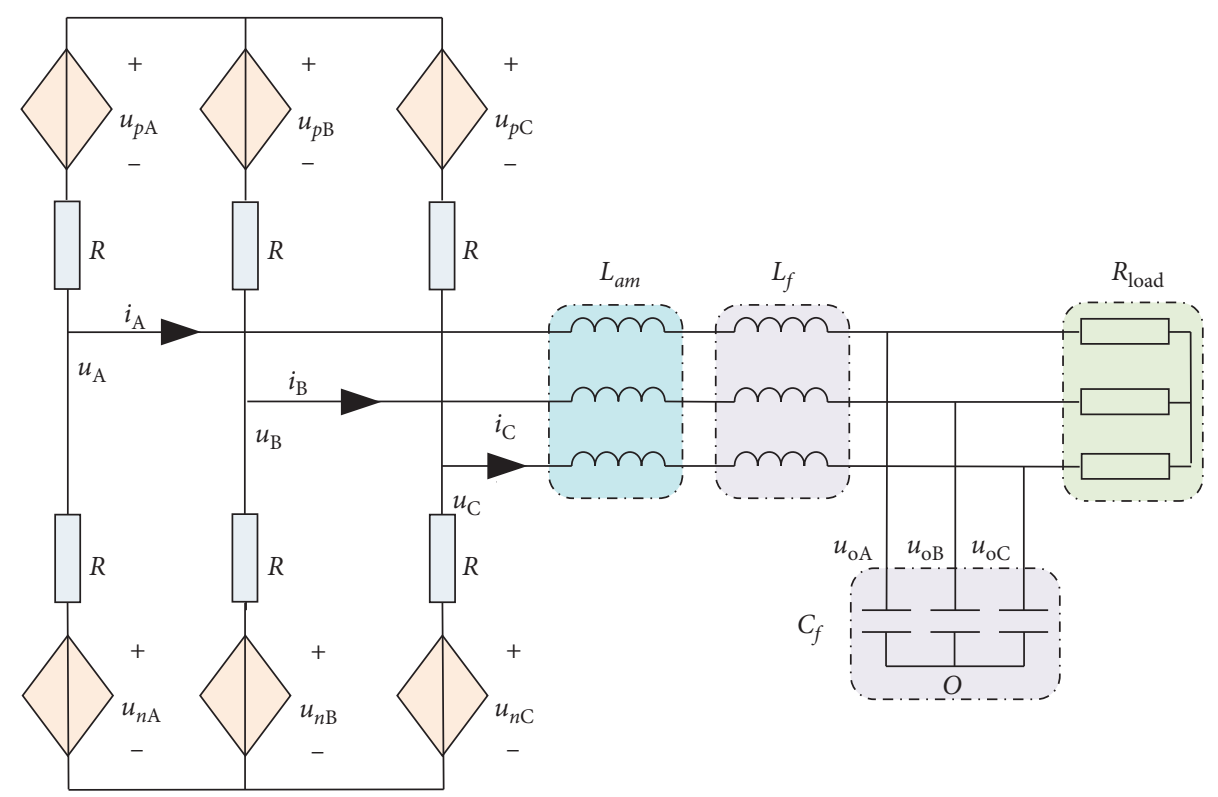

Figure 5: System equivalent circuit in the islanded mode.

\section{Design of System Output Controller}

In order to ensure the stable operation of MMC-MG in the islanded mode, the double-closed-loop control of voltage and current is adopted on the inverter to provide voltage and frequency support. The outer voltage loop determines the reference value of the instruction current and stabilizes the AC side voltage of the inverter. The inner current loop controls the current according to the instruction current to realize the fast tracking.

5.1. Sliding Mode Controller Design. Firstly, an outer voltage loop controller is designed to determine $i_{d}^{*}$ and $i_{q}^{*}$. Then, the SMC is adopted in the current inner loop controller to resist the influence of parameter perturbation and external disturbance on the feedback linearization model and improve the robustness of the system. Define the system tracking error as follows:

$$
\left[\begin{array}{l}
e_{1} \\
e_{2}
\end{array}\right]=\left[\begin{array}{c}
y_{1}^{*}-y_{1} \\
y_{2}^{*}-y_{2}
\end{array}\right]=\left[\begin{array}{c}
i_{\mathrm{d}}^{*}-i_{\mathrm{d}} \\
i_{\mathrm{q}}^{*}-i_{\mathrm{q}}
\end{array}\right] .
$$

The control effect of the sliding mode controller is related to the selection of the sliding surface. The traditional nonlinear integral sliding surface is

$$
s_{i}=c_{i 1} e_{i}-c_{i 2} \int_{0}^{t} e_{i} d \tau \text {. }
$$

In order to improve the robustness of the system, the function $f(\mathrm{t})$ is introduced to form the global integral sliding surface so that the initial state of the system is on the sliding surface, eliminating the arrival process. The system global integral sliding surface is

$$
s_{i}=c_{i 1} e_{i}-c_{i 2} \int_{0}^{t} e_{i} d \tau-f_{i}(t)
$$

where $f_{i}(t)=e_{i}(0) e_{i}^{-p t}, p>0$, and $c_{i 1}$ and $c_{i 2}$ are the coefficient of SMC.

According to the reaching law of the sliding mode, in order to reduce the system chattering, the reaching law is

$$
\dot{s}_{i}=-k_{i} s_{i}-\varepsilon_{i} \operatorname{sat}\left(s_{i}\right),
$$

where $\operatorname{sat}(s)$ is a saturation function, $k_{i}$ and $\varepsilon_{i}$ are the reaching law coefficients, and $k_{i}>0$ and $\varepsilon_{i}>0$. The existence of the boundary layer $\Delta$ makes sat $(s)$ satisfy

$$
\operatorname{sat}(s)= \begin{cases}1 & s>\Delta \\ k s & |s| \leq \Delta \quad k=\frac{1}{\Delta} . \\ -1 & s>\Delta\end{cases}
$$

Derivation of (19) is

$$
\dot{s}_{i}=c_{i 1} \dot{e}_{i}-c_{i 2} e_{i}+e_{i}(0) p e_{i}^{-p t} .
$$

Combining (17), (20), and (22), we obtain

$$
v_{i}=\frac{\left[c_{i 2} e_{i}-k_{i} s_{i}-\varepsilon_{i} \operatorname{sat}\left(s_{i}\right)-e_{i}(0) p e_{i}^{-p t}\right]}{c_{i 1}} .
$$

In this system, the input variables can be written as follows:

$$
\left\{\begin{array}{l}
v_{1}=\frac{\left[k_{1} s_{1}+\varepsilon_{1} \operatorname{sat}\left(s_{1}\right)+e_{1}(0) p e_{1}^{-p t}-c_{12} e_{1}\right]}{c_{11}}, \\
v_{2}=\frac{\left[k_{2} s_{2}+\varepsilon_{2} \operatorname{sat}\left(s_{2}\right)+e_{2}(0) p e_{2}^{-p t}-c_{22} e_{2}\right]}{c_{21}} .
\end{array}\right.
$$

Combining (24) and (16), the output control amount after system feedback linearization can be written as follows: 


$$
\left\{\begin{array}{l}
u_{\mathrm{d}}=u_{\mathrm{od}}+L_{m} v_{1}+R i_{\mathrm{d}} / 2-\omega_{0} L_{m} i_{\mathrm{q}} \\
u_{\mathrm{q}}=u_{\mathrm{oq}}+L_{m} v_{2}+R i_{\mathrm{q}} / 2+\omega_{0} L_{m} i_{\mathrm{d}} .
\end{array}\right.
$$

5.2. Voltage Fluctuation Compensation Controller Design. Affected by the arm current, there is still a small deviation in the GM DC-link voltage under the control of HESS. The superposition of the deviations makes the output voltage of the system include DC and fundamental frequency deviation components. Firstly, the influence of the deviation under the closed-loop on the system control is analyzed. Let $\Delta u_{\mathrm{pA}}=a$ and the rest be 0 . Based on (2) and (3), the output voltage deviation caused by $a$ is superimposed on the $u_{\text {od }}$ and $u_{\text {oq }}$ after dq0 transformation can be given by

$$
\left\{\begin{array}{l}
u_{\mathrm{zd}}=\frac{1}{2 \sqrt{3}} a \sin \left(\alpha+\frac{\pi}{3}\right) \\
u_{\mathrm{zq}}=\frac{1}{2 \sqrt{3}} a \sin \left(\alpha-\frac{\pi}{6}\right) \\
u_{\mathrm{sd}}=-\frac{3+\sqrt{3}}{24} a M \sin 2 \alpha \\
u_{\mathrm{sq}}=\frac{\sqrt{3}}{8} a M \sin \left(2 \alpha+\frac{\pi}{3}\right)-\frac{3}{16} a M
\end{array}\right.
$$

It means that the DC and fundamental frequency deviation components are converted to fundamental frequency component and double-frequency component by $\mathrm{dq} 0$ transformation.

Therefore, it is necessary to modify the SMC controller and suppress $\Delta u_{p \mathrm{X}}$ and $\Delta u_{n \mathrm{x}}$ influences on the output voltage by voltage fluctuation compensation (VFC) controller. The VFC controller is shown in Figure 6.

$\Delta u_{\mathrm{d}}$ and $\Delta u_{\mathrm{q}}$ are the corrections; then, the actual output of the controller is

$$
\left\{\begin{array}{l}
u_{\mathrm{d}-\mathrm{ref}}=u_{\mathrm{d}}+\Delta u_{\mathrm{d}} \\
u_{\mathrm{q}-\mathrm{ref}}=u_{\mathrm{q}}+\Delta u_{\mathrm{q}} .
\end{array}\right.
$$

Combining (17), (25), and (27), we can obtain the system output voltage control block diagram, as shown in Figure 7.

\section{Results and Discussion}

In order to validate the effectiveness of the HESS control strategy and MMC-MG output voltage control strategy, a simulation mode of MMC-MG was built in Matlab/Simulink. The MMC-MG configuration is given in Figure 1. The HESS configuration is given in Figure 3. The NO. of GMs in each arm is $N=4$, the arm inductor $L=1 \mathrm{mH}$, and the $\mathrm{HC}$ capacitor $C=4400 \mu \mathrm{F}$. GM DC-link voltage $u_{\mathrm{dc}}^{*}=160 \mathrm{~V}$.

6.1. Simulation Verification of HESS. A PV battery is used as the RES of GM, and a SC and battery which are used to form a HESS. For comparative analysis, the simulation models of

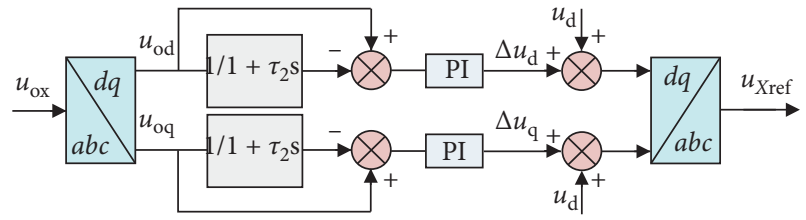

FIGURE 6: Block diagram of voltage suppression control.

PV DC power generation, PV inverter, nine-level cascaded $\mathrm{H}$-bridge $\mathrm{PV}$ inverter, and MMC-MG were established in turn. The PV simulation parameters are shown in Table 2.

The simulation results of PV DC-link voltages with different topologies under different solar incident irradiances are shown in Figure 8. $a$ is the output voltage of PV DC power generation, $b$ is the DC-link voltage of PV inverter, $c$ is the DC-link voltage of cascaded $\mathrm{H}$-bridge $\mathrm{PV}$ inverter, and $d$ is the DC-link voltage of GM.

It can be seen from the graph that when the solar radiation intensity changes at $1 \mathrm{~s}, 2 \mathrm{~s}, 3 \mathrm{~s}$, and $4 \mathrm{~s}$, the voltage $a$, $b$, and $c$ have the same trend and the amplitude fluctuation are small, while voltage amplitude of $d$ fluctuates greatly. This shows that, under the same solar radiation intensity conditions, due to the series structure of MMC-MG, the voltage fluctuation of GM is large.

The HESS is connected to the GM DC link to suppress the DC voltage fluctuation caused by the change of solar radiation intensity. Then, design the energy storage converter controller according to Figure 4. Under the HESS control, the GM DC-link voltages $u_{\mathrm{dc}}$ is shown in Figure 9. The amplitude fluctuation is small and the output voltage is stable when the solar radiation intensity changes. The control strategy can achieve good voltage stability control effect of GM DC-link.

The battery output current and the power $P_{\mathrm{G}}-P_{\mathrm{PV}}$ are shown in Figure 10(a). The SC output current is shown in Figure 10(b). It can be seen that the trend of $i_{b}$ is similar to that of $P_{\mathrm{G}}-P_{\mathrm{PV}}$, and the trend of $i_{\mathrm{sc}}$ is similar to that of highfrequency component of $P_{\mathrm{G}}-P_{\mathrm{PV}}$, which further reflects the difference and complementarities of battery and SC in energy balance. Besides, the SC is in frequent charge discharge state switching, while the battery state is relatively stable.

6.2. Simulation Verification of SMC. The HESS is used to ensure that the GM DC-link voltage is stable at $160 \mathrm{~V}$ under varying solar incident irradiances. Design the system output voltage controller according to Figure 7, design SMC according to (24), and design SFL according to (25). When MMC-MG is in the islanded mode, the output voltage and current of the system inverter is shown in Figures 11-15.

Figures 11(a) and 11(b) show the root mean square (RMS) of the line voltage and phase current. The load increases at $1 \mathrm{~s}, 2 \mathrm{~s}$, and $5 \mathrm{~s}$ and decreases at $3 \mathrm{~s}$ and $4 \mathrm{~s}$. As can be seen from the graph, the line voltage is adjusted rapidly after a small fluctuation to maintain the voltage stability, and the output phase current changes with the change of the load. 


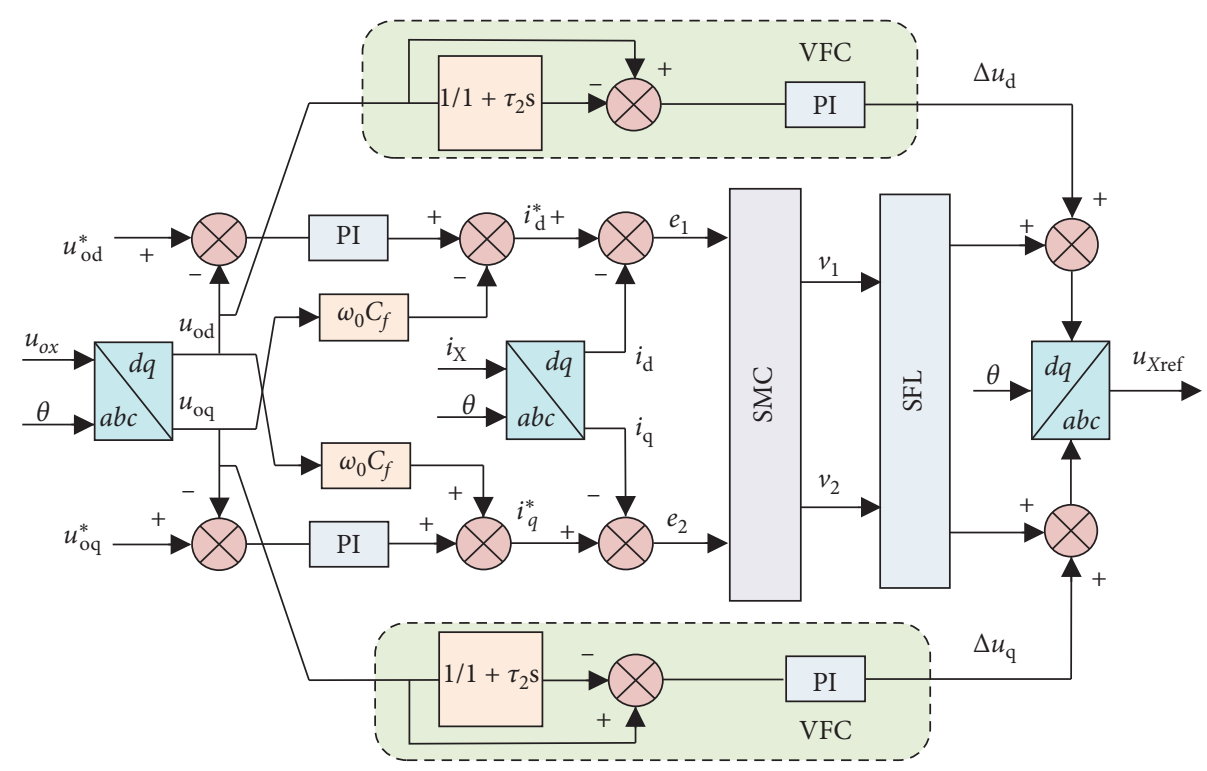

FIGURE 7: Block diagram of the system output voltage control.

TABLE 2: PV parameters.

\begin{tabular}{lc}
\hline Parameter & Value \\
\hline PV maximum power point voltage $(\mathrm{V})$ & 48 \\
PV maximum power point voltage $(\mathrm{A})$ & 10.2 \\
PV open circuit voltage $(\mathrm{V})$ & 56 \\
PV short circuit current $(\mathrm{A})$ & 10.8 \\
Temperature $\left({ }^{\circ} \mathrm{C}\right)$ & 25 \\
\hline
\end{tabular}

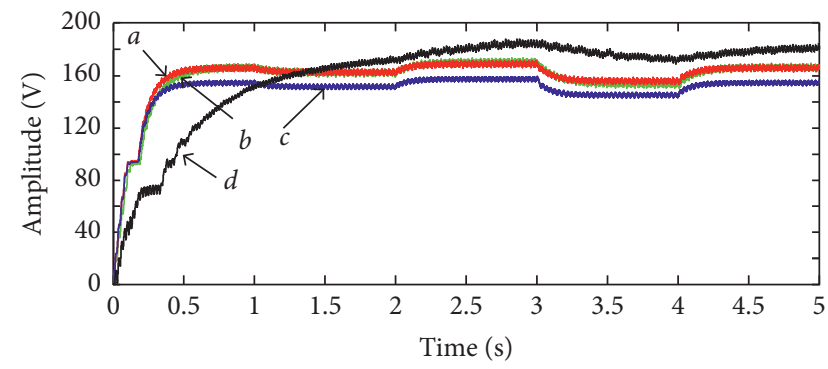

FIGURE 8: PV DC-link voltages.

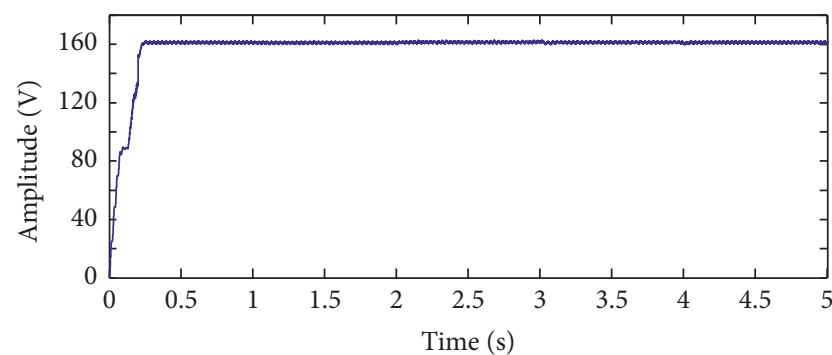

FIGURE 9: $u_{\mathrm{dc}}$ under HESS compensates.

Figures 12(a) and 12(b) show the voltage and current of system when the load changes from $14 \Omega$ to $10 \Omega$ at $2 \mathrm{~s}$. When the load increases, the line voltage amplitude recovers after a slight fluctuation, and the phase current increases.
Figure 13 shows the output voltage frequency. In case of sudden load change, the output frequency deviation is always within $\pm 0.2 \mathrm{~Hz}$, which meets the frequency requirement of the power grid.

Design the VFC controller according to Figure 6. The DC component of line voltage is shown in Figure 14. When the VFC strategy is applied to the system at $0.5 \mathrm{~s}$, the DC component is suppressed. At the moment of load switching from $1 \mathrm{~s}$ to $5 \mathrm{~s}$, the DC component changes abruptly, but decreases rapidly under the control effect. This strategy can achieve good DC component suppression effect of output line voltage.

Fundamental frequency deviation $u_{S A B}$ is the same as the frequency of fundamental frequency component of $u_{\mathrm{AB}}$, with different phase and smaller amplitude. In order to verify the suppression effect of VFC strategy on $u_{S A B}, u_{S B C}$, and $u_{\text {SCA }}$ more intuitively, the FFT analysis of $u_{\text {od }}$ after dq0 transformation of the line voltage before and after the VFC control is carried out.

As shown in Figure 15(a), without VFC control, the fundamental component $u_{z \mathrm{~d}}$ caused by the DC component of the line voltage is $0.98 \mathrm{~V}$, and the second harmonic component $u_{s \mathrm{~d}}$ caused by the fundamental frequency deviation component of the line voltage is $0.26 \mathrm{~V}$. As shown in Figure 15(b), with VFC control, the amplitude of $u_{z \mathrm{~d}}$ and $u_{s \mathrm{~d}}$ decreased to $0.2 \mathrm{~V}$ and $0.11 \mathrm{~V}$. It shows that the VFC strategy can achieve good fundamental frequency deviation suppression effect of line voltage.

Figure 16 compares the response curves of the current inner loop using traditional PI control and SMC. It can be seen from the graph that under the condition of sudden load changes, both control strategies can maintain the system output voltage stability, but the overshoot and adjustment time of the SMC are better than the PI control. It can be seen from Figures 11 to 15 that, under the condition of GM DClink voltage fluctuation and load mutation, the designed SMC controller has good anti-interference effect and can realize stable control of system output voltage. 


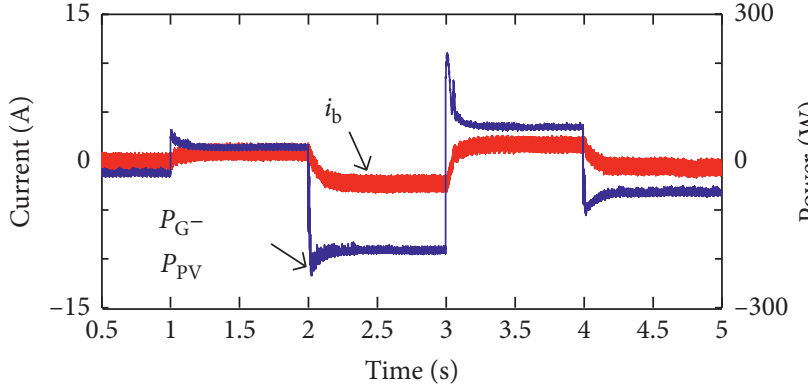

(a)

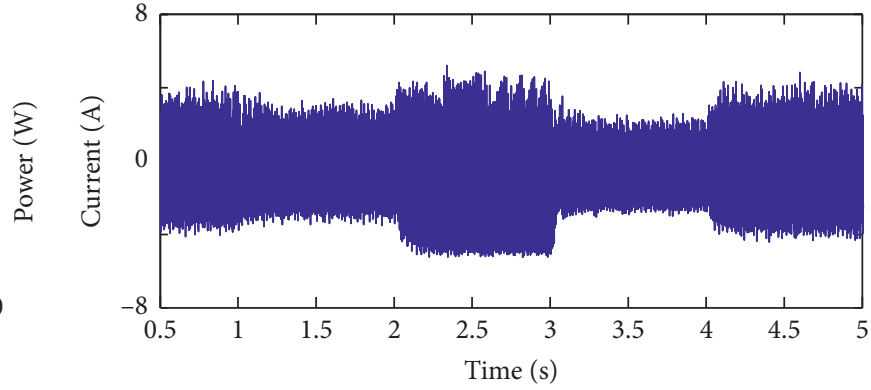

(b)

FIgURe 10: Battery output current, power $P_{\mathrm{G}}-P_{\mathrm{PV}}$, and SC output current.

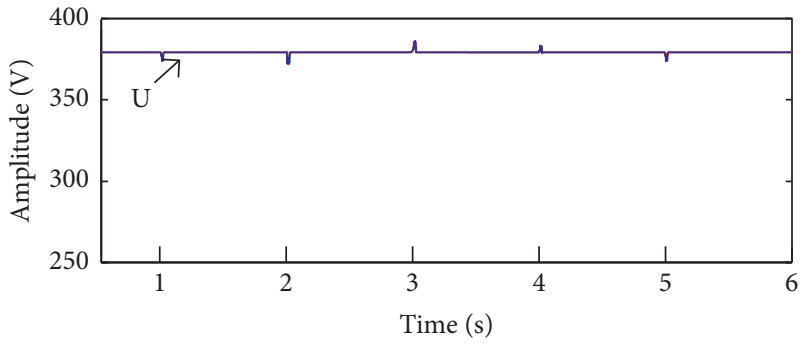

(a)

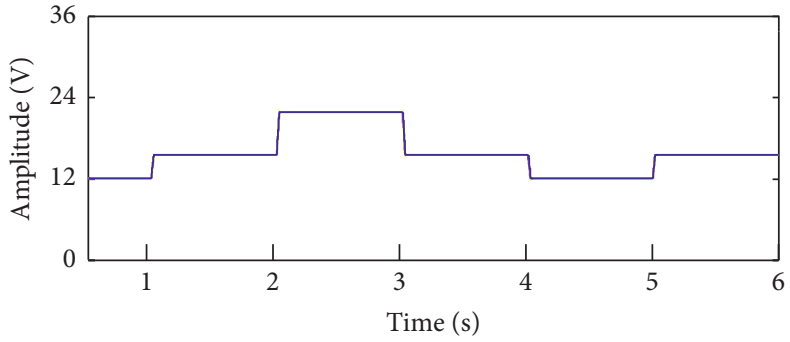

(b)

FIgURE 11: Line voltage and phase current RMS under time-varying load.

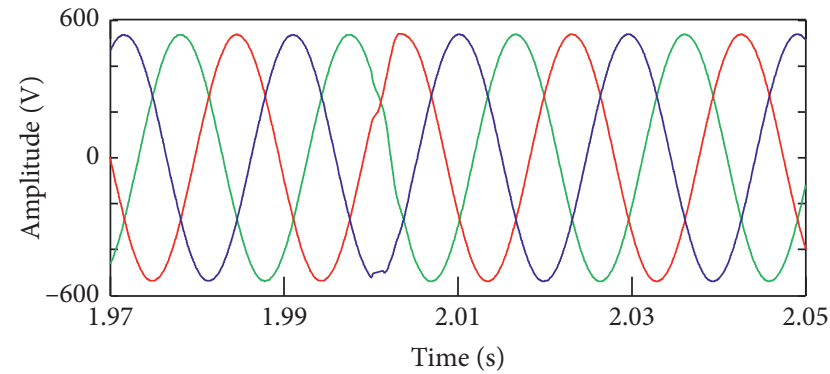

(a)

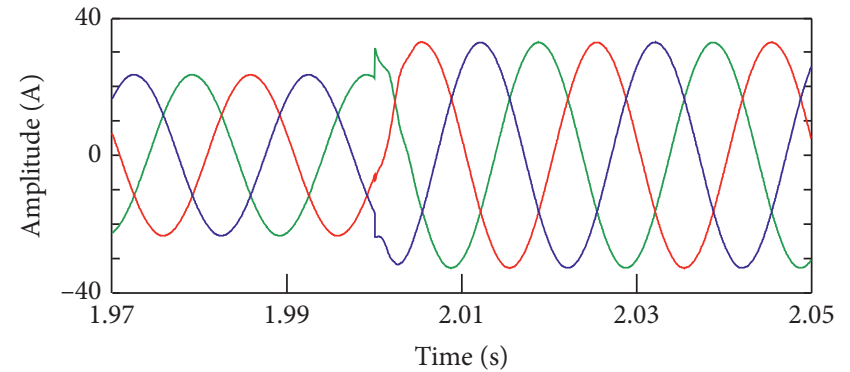

(b)

FIgURE 12: Line voltage and phase current at the moment of load change.

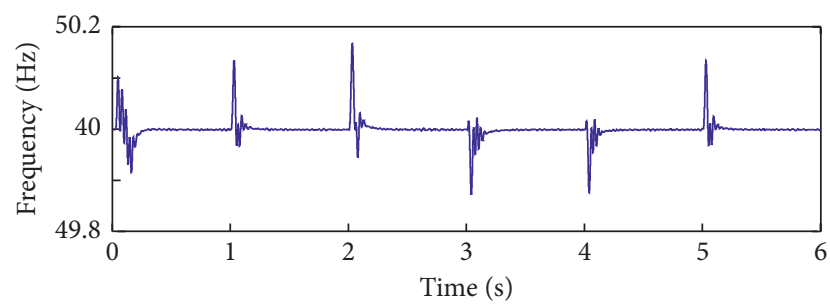

FIGURE 13: Voltage frequency under time-varying load.

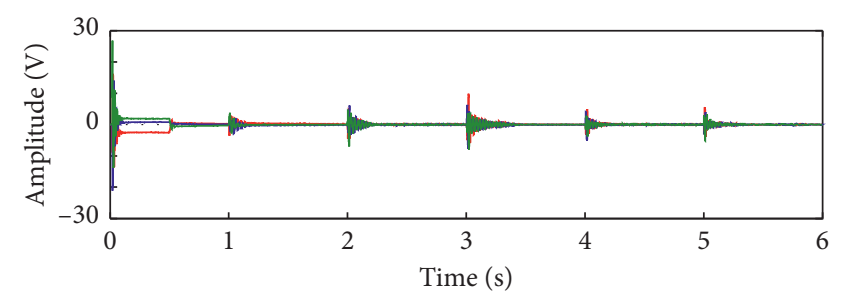

FIGURE 14: DC component of line voltage under time-varying load. 


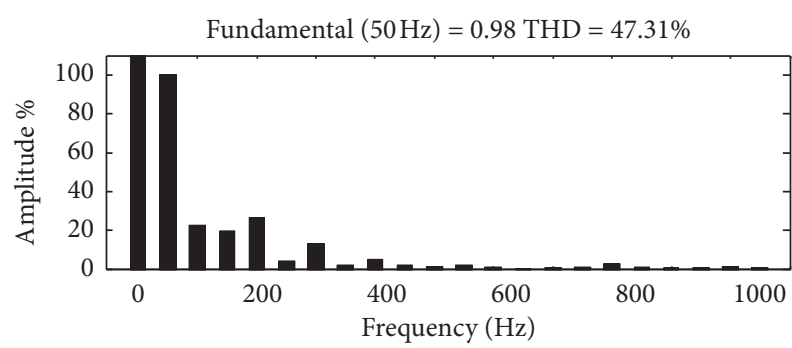

(a)

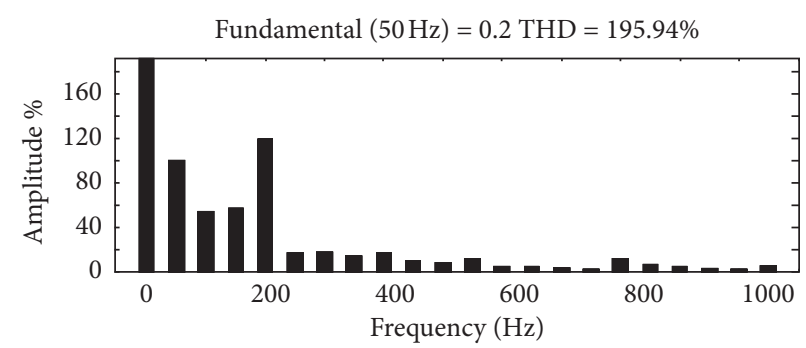

(b)

FIGURE 15: $\mathrm{u}_{\mathrm{od}}$ frequency analysis.

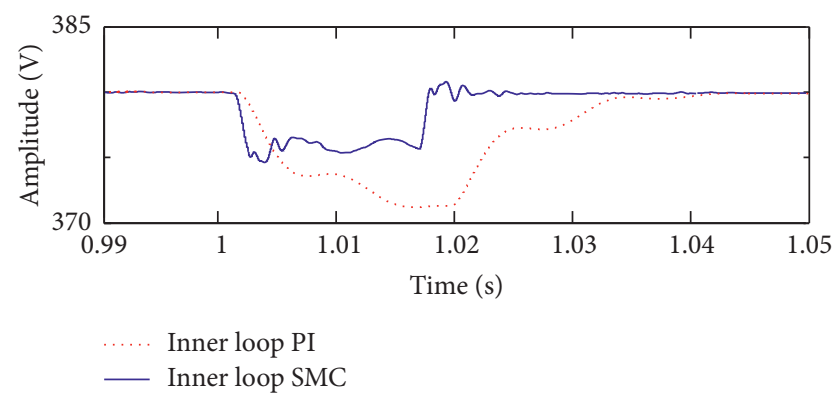

Figure 16: Control system response curve.

\section{Conclusions}

In this paper, the voltage fluctuation mechanism of GM DClink caused by the randomness of RES output power is analyzed, and the GM DC-link voltage stability control is realized by HESS control. The sliding mode controller based on GM voltage fluctuation compensation is designed to realize the stable control of the system output voltage in the islanded mode.

The simulation results show that the HESS control strategy can effectively suppress the GM DC-link voltage fluctuation caused by the RES output power variation. The sliding mode controller has fast response speed and strong antidisturbance capability than the PI controller. Under the condition of sudden load change, the system voltage regulation speed is fast, the amplitude change is small, and the frequency fluctuation is $\pm 0.2 \mathrm{~Hz}$. The VFC controller can effectively suppress DC components and fundamental deviation components of the system output voltage and improve the power quality. Hence, the proposed control strategy has a good adaptability to MMC-MG.

\section{Nomenclature}

$\begin{array}{ll}\text { MG: } & \text { Microgrid } \\ \text { DG: } & \text { Distributed generation } \\ \text { RES: } & \text { Renewable energy sources } \\ \text { GM: } & \text { Generating module } \\ \text { MS: } & \text { Microsources } \\ \text { HESS: } & \text { Hybrid energy storage system } \\ \text { SC: } & \text { Supercapacitor } \\ \text { SMC: } & \text { Sliding mode control }\end{array}$

VFC: Voltage fluctuation compensation

HC: Half-bridge converter

STS: $\quad$ Static transfer switch

SFL: $\quad$ State feedback linearization

$u_{Z \mathrm{AB}}: \quad$ DC component of $u_{\mathrm{AB}}(\mathrm{V})$

$u_{S A B}: \quad$ Fundamental deviation of $u_{\mathrm{AB}}(\mathrm{V})$

$u_{\mathrm{dc}}^{*}: \quad$ Voltage reference value of GM DC link (V)

$u_{p \mathrm{X}_{i}}, u_{n \mathrm{X}_{i}}: i$ th GM output voltage of positive and negative arm in $X$ phase (V)

$\Delta u_{p \mathrm{X}_{i}}: \quad$ Deviation of $i$ th GM DC-link voltage in $X$ phase positive arm (V)

$\Delta u_{n \mathrm{X}_{i}}$ : Deviation of $i$ th GM DC-link voltage in $X$ phase negative arm (V)

$i_{\mathrm{g}}: \quad$ Output current of microsource $(\mathrm{A})$

$i_{\mathrm{c}}$ : $\quad$ Charge current of $C(\mathrm{~A})$

$i_{\mathrm{m}}$ : Input current of half-bridge converter (A)

$i_{p \mathrm{X}}, i_{n \mathrm{X}}$ : Positive arm and negative arm currents of $X$ phase (A)

$P_{\mathrm{M}}, P_{\mathrm{G}}: \quad$ Microsource output power and GM output power (A)

$u_{\mathrm{sc}}, i_{\mathrm{sc}}: \quad$ SC voltage and output current $(\mathrm{V}, \mathrm{A})$

$u_{\mathrm{b}}, i_{\mathrm{b}}$ : Battery voltage and output current $(\mathrm{V}, \mathrm{A})$

$P_{\mathrm{F}}: \quad$ Absorption energy of HESS (W)

$u_{\text {sc-max }}: \quad$ Maximum voltage of SC (V)

$u_{\text {sc-min: }}$ Minimum voltage of SC (V)

$u_{\mathrm{sc}-\mathrm{h}}, u_{\mathrm{sc}-1}$ : Upper limit value and lower limit value of SC voltage controller (V)

$u_{\mathrm{X}}, i_{\mathrm{X}}$ : Output current and output voltage of phase $X(\mathrm{~V}$, A)

$u_{\mathrm{oX}}: \quad$ Filter capacitor voltage of phase $X(\mathrm{~V})$

$R: \quad$ Arm equivalent resistance $(\Omega)$

$u_{\mathrm{d}}, i_{\mathrm{d}}: \quad$ Active components of the phase voltage and current $(\mathrm{V}, \mathrm{A})$

$u_{\mathrm{q}}, i_{\mathrm{q}}$ : Reactive components of the phase voltage and current $(\mathrm{V}, \mathrm{A})$

$u_{\mathrm{od}}, i_{\mathrm{od}}$ : Active components of the capacitor voltage and load current $(\mathrm{V}, \mathrm{A})$

$u_{\mathrm{oq}}, i_{\mathrm{oq}}$ : Reactive components of the capacitor voltage and load current (V, A).

\section{Data Availability}

The data used to support the findings of this study are available from the corresponding author upon request. 


\section{Conflicts of Interest}

The authors declare that there are no conflicts of interest regarding the publication of this paper.

\section{Acknowledgments}

This research was funded by the National Natural Science Foundation of China, Grant no. 51967011.

\section{References}

[1] R. H. Lasseter and P. Paigi, "Microgrid: a conceptual solution," in Proceedings of the 2004 IEEE 35th Annual Power Electronics Specialists Conference (IEEE Cat. No.04CH37551), pp. 4285-4290, Aachen, Germany, June 2004.

[2] N. Hatziargyriou, H. Asano, R. Iravani, and C. Marnay, "Microgrids," IEEE Power and Energy Magazine, vol. 5, no. 4, pp. 78-94, 2007.

[3] E. J. Ng and R. A. El-Shatshat, "Multi-microgrid control systems (MMCS)," in Proceedings of the IEEE PES General Meeting, pp. 1-6, Providence, RI, USA, July 2010.

[4] D. Wu, F. Tang, T. Dragicevic, J. C. Vasquez, and J. M. Guerrero, "A control architecture to coordinate renewable energy sources and energy storage systems in islanded microgrids," IEEE Transactions on Smart Grid, vol. 6, no. 3, pp. 1156-1166, 2015.

[5] J. W. Simpson-Porco, Q. Shafiee, F. Dörfler, J. C. Vasquez, J. M. Guerrero, and F. Bullo, "Secondary frequency and voltage control of islanded microgrids via distributed averaging," IEEE Transactions on Industrial Electronics, vol. 62, no. 11, pp. 7025-7038, 2015.

[6] Y. Xu, H. Sun, W. Gu, Y. Xu, and Z. Li, "Optimal distributed control for secondary frequency and voltage regulation in an islanded microgrid," IEEE Transactions on Industrial Informatics, vol. 15, no. 1, pp. 225-235, 2019.

[7] X. Tang, X. Hu, N. Li, W. Deng, and G. Zhang, "A novel frequency and voltage control method for islanded microgrid based on multienergy storages," IEEE Transactions on Smart Grid, vol. 7, no. 1, pp. 410-419, 2016.

[8] S. Adhikari and F. Li, "Coordinated V-f and P-Q control of solar photovoltaic generators with MPPT and battery storage in microgrids," IEEE Transactions on Smart Grid, vol. 5, no. 3, pp. 1270-1281, 2014

[9] X. G. Wang, S. Xue, and X. Y. Li, "Analysis of output characteristics of a microgrid based on modular multilevel converter half-bridge series structure," Transactions of China Electrotechnical Society, vol. 34, no. 10, pp. 2130-2140, 2019.

[10] B. V. Solanki, K. Bhattacharya, and C. A. Cañizares, "A sustainable energy management system for isolated microgrids," IEEE Transactions on Sustainable Energy, vol. 8, no. 4, pp. 1507-1517, 2017.

[11] Y. Li, Z. Yang, G. Li, D. Zhao, and W. Tian, "Optimal scheduling of an isolated microgrid with battery storage considering load and renewable generation uncertainties," IEEE Transactions on Industrial Electronics, vol. 66, no. 2, pp. 1565-1575, 2019.

[12] T. Morstyn, A. V. Savkin, B. Hredzak, and V. G. Agelidis, "Multi-agent sliding mode control for state of charge balancing between battery energy storage systems distributed in a DC microgrid," IEEE Transactions on Smart Grid, vol. 9, no. 5, pp. 4735-4743, 2018.

[13] S. Kotra and M. K. Mishra, "A supervisory power management system for a hybrid microgrid with HESS," IEEE
Transactions on Industrial Electronics, vol. 64, no. 5, pp. 3640-3649, 2017.

[14] A. Anzalchi, M. M. Pour, and A. Sarwat, "A combinatorial approach for addressing intermittency and providing inertial response in a grid-connected photovoltaic system," in Proceedings of the 2016 IEEE Power and Energy Society General Meeting (PESGM), pp. 1-5, Boston, MA, USA, July 2016.

[15] J. Fang, Y. Tang, H. Li, and X. Li, "A battery/ultracapacitor hybrid energy storage system for implementing the power management of virtual synchronous generators," IEEE Transactions on Power Electronics, vol. 33, no. 4, pp. 28202824, 2018.

[16] Q. Tabart, I. Vechiu, A. Etxeberria, and S. Bacha, "Hybrid energy storage system microgrids integration for power quality improvement using four-leg three-level NPC inverter and second-order sliding mode control," IEEE Transactions on Industrial Electronics, vol. 65, no. 1, pp. 424-435, 2018.

[17] T. Kerdphol, F. S. Rahman, Y. Mitani, M. Watanabe, and S. Kufeoglu, "Robust virtual inertia control of an islanded microgrid considering high penetration of renewable energy," IEEE Access, vol. 6, pp. 625-636, 2018.

[18] H. M. Ibrahim, M. S. El Moursi, and P.-H. Huang, "Adaptive roles of islanded microgrid components for voltage and frequency transient responses enhancement," IEEE Transactions on Industrial Informatics, vol. 11, no. 6, pp. 1298-1312, 2015.

[19] M. B. Delghavi and A. Yazdani, "Sliding-mode control of AC voltages and currents of dispatchable distributed energy resources in master-slave-organized inverter-based microgrids," IEEE Transactions on Smart Grid, vol. 10, no. 1, pp. 980-991, 2019.

[20] M. Cucuzzella, G. P. Incremona, and A. Ferrara, "Design of robust higher order sliding mode control for microgrids," IEEE Journal on Emerging and Selected Topics in Circuits and Systems, vol. 5, no. 3, pp. 393-401, 2015.

[21] W. Uddin, K. Zeb, M. A. Adil Khan et al., "Control of output and circulating current of modular multilevel converter using a sliding mode approach," Energies, vol. 12 , no. 21, p. 4084, 2019.

[22] A. Khaligh and Z. Zhihao Li, "Battery, ultracapacitor, fuel cell, and hybrid energy storage systems for electric, hybrid electric, fuel cell, and plug-in hybrid electric vehicles: state of the art," IEEE Transactions on Vehicular Technology, vol. 59, no. 6, pp. 2806-2814, 2010.

[23] Y. Zhang, L. Guo, H. J. Jia, and C. S. Wang, "An energy management method of hybrid energy storage system based on smoothing control," Automation of Electric Power Systems, vol. 36, no. 16, pp. 36-41, 2012. 